Article

\title{
Graphene-Gold Nanostructures Hybrid Composites Screen-Printed Electrode for the Sensitive Electrochemical Detection of Vancomycin
}

\author{
Adrian Blidar ${ }^{\dagger}$, Bogdan Feier $^{\dagger}$, Alexandra Pusta, Ana-Maria Drăgan and Cecilia Cristea ${ }^{*}$ \\ Department of Analytical Chemistry, "Iuliu Hațieganu" University of Medicine and Pharmacy, 4 Pasteur Street, \\ 400349 Cluj-Napoca, Romania; blidar.adrian@umfcluj.ro (A.B.); Feier.George@umfcluj.ro (B.F.); \\ alexandrapusta@gmail.com (A.P.); ana.drgn@yahoo.com (A.-M.D.) \\ * Correspondence: ccristea@umfcluj.ro \\ + These authors contributed equally to this work.
}

Received: 29 August 2019; Accepted: 8 October 2019; Published: 10 October 2019

\begin{abstract}
The most important and well-known glycopeptide antibiotic, vancomycin (VAN), is used for the treatment of severe bacterial infections like methicillin-resistant staphylococcal infections and endocarditis caused by enterococci and sepsis. Taking into account the problem of the development of antibiotic resistance as well as its toxicity, both correlated with the VAN concentration $\left(C_{\text {VAN }}\right)$ in biological samples, there is need for better and more accessible quantification methods for this antibiotic. Considering all of the above, herein, we present a simple electrochemical method for VAN sensing based on a hybrid graphene-gold nanostructure nanocomposite electrode, which allows double detection directly in the oxidation domain and also indirectly, in reduction, using the electro-active gold nanostructures as a probe to monitor the current changing due to the interaction between gold and VAN. The developed method was able to successfully detect VAN in the linear range of $1-100 \mu \mathrm{M}$ with a limit of detection (LOD) of $0.29 \mu \mathrm{M}$ for the direct approach and $0.5 \mu \mathrm{M}$ for the indirect one. The selectivity of the method was tested in the presence of other antibiotics and drugs. This method was successfully applied for the detection of VAN from human serum samples.
\end{abstract}

Keywords: vancomycin; graphene; screen printed electrodes; antibiotic detection; gold nanostructures

\section{Introduction}

The most important and well-known glycopeptide antibiotic, vancomycin (VAN) (Figure 1), is used for the treatment of severe bacterial infections like methicillin-resistant staphylococcal infections and endocarditis caused by enterococci, bone, lower respiratory tract, soft tissue infections and sepsis. It is active systemically only when administered parenterally with a single intravenous dose of $1 \mathrm{~g}$ producing plasma concentrations of 15 to $30 \mu \mathrm{g} / \mathrm{mL}$ or approximately 10 to $20 \mu \mathrm{M}$ [1].

VAN is active against Gram-positive bacteria resistant to other antibiotics, like enterococci, streptococci and staphylococci, making it often the last resort for the antibacterial treatment of complicated cases, involving antibiotic-resistant bacteria. Other advantages of VAN, compared to relatively newly approved molecules of antibiotics with similar indications, are its familiarity and better understanding by the medical staff and its lower price [1,2]. 


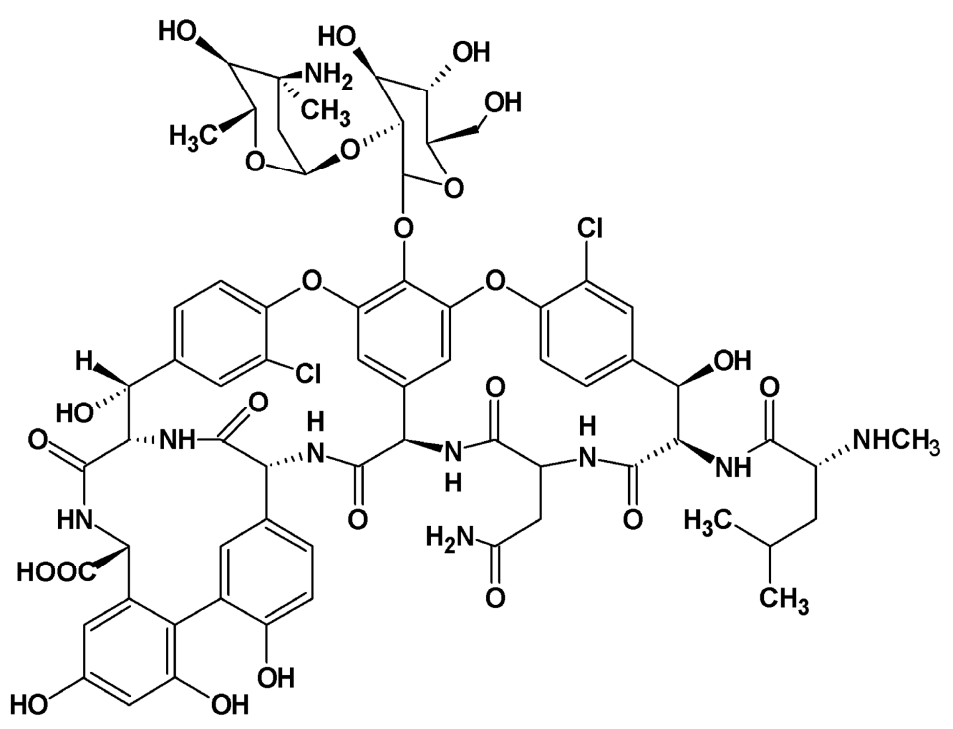

Figure 1. The chemical structure of VAN.

As already reported, the treatment with VAN is associated with a relatively high occurrence of nephrotoxicity and ototoxicity. Another major concern is related to the spread of bacterial resistance to VAN [1,2].

Nowadays, it is known that the widespread use of antibiotics can result in the presence of residues in the environment and in food that can lead to health problems. Antimicrobial resistance has been defined by World Health Organization (WHO) as the "ability of a microorganism (like bacteria, viruses, and some parasites) to stop an antimicrobial (such as antibiotics, antivirals and antimalarials) from working against it" and it generates higher costs of health care [3,4]. This problem is even more disturbing when talking about antibiotics of last resort, such as VAN.

WHO also has endorsed a global action plan on this topic, recommending an urgent improvement in the surveillance of the use of antibiotics involving the therapeutic monitoring of antibiotics in order to adjust the dosage for an efficient treatment. This action, to be successful, needs new, rapid and more accessible detection methods for the determination of antibiotics from different and complex matrices [3-5].

Considering the importance of VAN concentration $\left(C_{\mathrm{VAN}}\right)$ in biological samples, levels too high being correlated with severe adverse effects and levels too low with the development of antibiotic resistance, along with the large number of patients treated with VAN, the frequent quantification of blood levels in therapeutic drug monitoring, and the cost of the available methods, the need for better and more accessible quantification methods for VAN is of high interest.

In general, antibiotics have been detected through microbiological-based techniques, which rely on the growth inhibition of Bacillus stearothermophilus [6]. These tests often present a long analysis time and a lack of selectivity, not being able sometimes to distinguish even among different antibiotic classes. Nowadays, the most used methods for the detection of VAN are the chromatographic, spectrophotometric (after derivatization) and immunochemical methods [7].

The liquid chromatography analyses are usually preceded by sample preparation and extraction, leading to the elimination of interferences and preconcentration of target analytes. Even though all these methods are sensitive and selective, they present some disadvantages: they are expensive and time-consuming and require complex analytical equipment, extensive sample handling, use of expensive and pollutant solvents and very qualified staff, and they do not allow field analyses, making the development of new sensors necessary to overcome these limitations $[7,8]$.

The electrochemical methods can overcome these limitations, especially when considering screen printed electrodes (SPE), which are mass produced, inexpensive, meant for single use, and sterilizable, making them very suitable for use in clinical monitoring in point-of-care devices [9]. 
In this regard, the direct electrochemical detection of VAN was recently reported $[9,10]$. Considering its large molecule and the need of analysis from complex matrices, a more complex approach for the direct electrochemical detection, involving the development and use of a complex electrochemical fingerprint of VAN, was recently reported [9].

For the direct electrochemical detection of VAN there is a need for new electrochemical platforms, with a higher sensitivity towards this molecule. This is because in the data reported in the literature $[9,10]$, the combination of graphene and gold nanostructures (AuNSs) or gold nanoparticles (AuNPs) can represent a viable alternative due to the synergic electrocatalytic effect of graphene and AuNSs towards electrooxidation of VAN, but also due to the interaction between the AuNSs and VAN, which is usable in the cathodic domain $[9,10]$.

Graphene is a single-atom-thick sheet consisting of $s p 2$ hybridized carbon atoms, with unique properties such as large surface area, high electronic conductivity and rapid heterogeneous electron transfer, making it a great and intensively used as electrode material [11,12]. The use of graphene is also sustained by the reported affinity of VAN towards graphene [13-15].

Similarly, AuNSs are an attractive nanomaterial for the fabrication of electroanalytical platforms because of their large specific surface area, good biocompatibility, strong binding ability, and excellent conductivity [14]. They are also attractive due to the previously reported interaction between VAN and different forms of $\mathrm{Au}$ (AuNPs or ionic $\mathrm{AuCl}^{-}$), which can be exploited in electroanalysis [9,16-18]. VAN can interact with $\mathrm{HAuCl}_{4}$, leading to the formation of VAN-caped AuNPs, with enhanced antibacterial properties [16-18], but can also interact with already synthesized AuNPs. This affinity of VAN for AuNSs, together with their special electrochemical properties can be exploited for analytical purposes, in the development of methods for VAN quantification.

The use of the graphene-AuNSs hybrid for vancomycin detection is an original approach, which allows a double detection method, on one sensing surface.

Considering all of the above, herein, we present a simple electrochemical method for VAN sensing based on a hybrid graphene-AuNSs nanocomposite electrode, which allows a double detection, in one analysis, directly, in the oxidation domain and also indirectly, in reduction, using the electro-active AuNSs as probe to monitor the current changing due to the interaction between Au and VAN.

The developed method was able to successfully detect VAN in the linear range of 1-100 $\mu \mathrm{M}$ with a LOD of $0.29 \mu \mathrm{M}$ for the direct approach and $0.5 \mu \mathrm{M}$ for the indirect one. The selectivity of the method was tested in the presence of other antibiotics and drugs. This method was successfully applied for the detection of VAN from human serum samples.

\section{Materials and Methods}

\subsection{Materials}

All chemicals were of analytical grade and were used as received without further purification. $\mathrm{HCl}, \mathrm{Na}_{2} \mathrm{HPO}_{4}, \mathrm{NaH}_{2} \mathrm{PO}_{4}, \mathrm{NaCl}, \mathrm{HAuCl}_{4}, \mathrm{HClO}_{4}, \mathrm{H}_{2} \mathrm{SO}_{4}$, ampicillin trihydrate (AMP), penicillin $\mathrm{V}$ potassium salt, penicillin $\mathrm{G}$ sodium salt, oxacillin sodium salt, amoxicillin, ascorbic acid, acetaminophen, and the human serum were purchased from Sigma-Aldrich (Saint Louis, MO, USA); cefalexin monohydrate (CFX) from Antibiotice SA (Iași, Romania); vancomycin sulfate from Linaris, Dossenheim, Germany; and gentamicin sulphate from Bioworld, Dublin, OH, USA. AuNPs of 15 and $50 \mathrm{~nm}$ were purchased from Nanovex Biotechnologies, Asturias, Spain. In order to test if the size of these AuNPs influences the VAN detection, these two dimensions were chosen as they are at extremes of the usual dimensions of AuNPs obtained through electrogeneration [19].

All solutions were prepared with ultrapure water (18.2 M $\Omega$, Millipore Simplicity, Burlington, MA, USA).

The supporting electrolyte used in this study was the solution of $0.05 \mathrm{~mol} \cdot \mathrm{L}^{-1}$ phosphate buffer saline (PBS) prepared with $\mathrm{Na}_{2} \mathrm{HPO}_{4}, \mathrm{NaH}_{2} \mathrm{PO}_{4}$ and $\mathrm{NaCl}$, adjusted to the mentioned values of $\mathrm{pH}$. 
All experiments were performed in compliance with the ethics guidelines of the "Iuliu Hatieganu" University of Medicine and Pharmacy, Cluj-Napoca, Romania.

\subsection{Instruments}

The electrochemical experiments were performed using an AUTOLAB PGSTAT 302N (Ecochemie, Utrecht, The Netherlands) equipped with the associated NOVA 1.10.4 software.

All the SPEs, with different working electrodes (carbon-based (C-SPE), carbon-based modified with graphene (G-SPE) and carbon-based modified with gold nanoparticles (AuNPs-C-SPE)) and with a silver pseudoreference and a carbon counter electrode were purchased from Dropsens (Asturias, Spain).

Spectrophotometric analyses were performed using a SPECORD 250 PLUS UV-VIS spectrophotometer (Analytik Jena AG, Jena, Germany), with $1 \mathrm{~cm}$ cells.

\subsection{Methods}

\subsubsection{Electrochemical Characterization}

Two electrochemical techniques, cyclic voltammetry (CV) and differential pulse voltammetry (DPV), were used for the electrochemical characterization of VAN behaviour on the modified surfaces.

$\mathrm{CV}$ experiments were performed in the potential window of $-0.2 \mathrm{~V}$ to $+1.2 \mathrm{~V}$, with a scan rate of $100 \mathrm{mV} / \mathrm{s}$.

The parameters used for the DPV technique were a pulse height $(\mathrm{PH})$ of $100 \mathrm{mV}$, a pulse width (PW) of $50 \mathrm{~ms}$, and a scan rate (SR) of $0.02 \mathrm{~V} \cdot \mathrm{s}^{-1}$ for the oxidation studies, the only difference for the reduction studies being a $\mathrm{PH}$ of $-100 \mathrm{mV}$.

\subsubsection{UV-VIS Characterization}

The UV-VIS studies were carried on recording the UV spectral scans between 200 and $350 \mathrm{~nm}$ and the VIS spectral scans between 350 and $850 \mathrm{~nm}$, with a $1 \mathrm{~nm}$ slit, $1 \mathrm{~nm}$ wave length step, and a speed of $5 \mathrm{~nm} \cdot \mathrm{s}^{-1}$.

\subsubsection{Functionalization of the Working Surface with AuNSs and AuNPs}

The modification of the SPEs with AuNSs and AuNPs was carried out employing two different approaches, one involving the electrogeneration of the AuNSs, onto the sensing surface and a second one involving a drop-casting protocol, using a suspension of AuNPs.

For the electrogeneration of AuNSs, a volume of $60 \mu \mathrm{L}$ of an aqueos solution of $0.6 \mathrm{mM} \mathrm{HAuCl}_{4}$ and $0.5 \mathrm{M} \mathrm{H}_{2} \mathrm{SO}_{4}$ was deposited on the SPE surface and two different electrochemical techniques were used, CV and chronoamperommetry (CA) [20]. For the CV technique, the potential was scanned in the window of $-0.2 \mathrm{~V}$ to $+1.2 \mathrm{~V}$, with a scan rate of $100 \mathrm{mV} / \mathrm{s}$, for a number of 10 cycles. For the CA technique, the main parameters used, potential and duration, are mentioned in the Results and Discussion section for each experiment.

For the drop-casting protocol, a volume of $10 \mu \mathrm{L}$ of an aqueous suspension of AuNPs, with a particle concentration of $6 \times 10^{11}$ particles $/ \mathrm{mL}$, was deposited on the SPE surface and left to dry at room temperature. This procedure was repeated up to five times, totaling a volume of $50 \mu \mathrm{L}$, in order to assess the influence of the amount and of the thickness of the modifier. AuNPS of two dimensions, 15 and $50 \mathrm{~nm}$, were used.

\subsubsection{Selectivity Tests and Real Samples Analysis}

The optimized method was tested for possible interferents, mainly other antibiotics (amoxicillin (AMX), penicillin V (PenV), penicillin $G($ PenG), oxacillin (OXA), cefalexin (CFX), gentamicin (GEN)), but also other drugs, like acetaminophen (ACP) and ascorbic acid (AAC), by testing the signal of VAN in the presence of the aforementioned interferents, in mixture solutions. 
The method was also tested on biological samples, more precisely commercially available human serum. The samples were spiked with different concentrations of VAN in the linear range of the method. Before the analysis, a volume of $1 \mathrm{~mL}$ of the spiked and unspiked samples was treated with $50 \mu \mathrm{L} \mathrm{HClO}_{4} 70 \%$ and centrifuged at $10000 \mathrm{rpm}$, for $10 \mathrm{~min}$, for protein precipitation. After that, the supernatant was diluted $1: 10$ with $0.1 \mathrm{M} \mathrm{H}_{2} \mathrm{SO}_{4}$ and tested.

\subsubsection{Statistics and Data Processing}

All analyses were performed in triplicate with the values presented in the text representing the average value. The calibration curves were constructed using the least squares regression method and the error bars presented represent the standard deviation obtained for the three analyses performed for each concentration.

The statistical treatment, data analysis and the creation of figures were performed using the Origin 8 software (OriginLab, Northampton, MA, USA).

For better visualization, all the DPV voltammograms presented here were baseline-corrected using the moving average filter included in the NOVA 1.10.4 software (window size 1), without affecting the results.

\section{Results}

\subsection{VAN Interaction with $A u$ and $A u N S$}

Continuing our previous work regarding VAN detection [9], we investigated VAN interaction with $\mathrm{Au}$ both with gold cations $\left(\mathrm{Au}^{3+}\right)$ and with different AuNSs and how these interactions could be exploited in an electroanalytical method for VAN detection.

\subsubsection{Electrochemical Studies}

CV tests were carried out on AuNPs-C-SPE and on C-SPE and G-SPE, modified in the lab with AuNS, electrochemically generated using $\mathrm{CV}$ from a solution of $\mathrm{HAuCl}_{4}$. For all three surfaces a single well-defined reduction peak, around $+0.5 \mathrm{~V}$, could be observed for the blank solution.

When the VAN-containing solutions were tested, a clear shift around $200 \mathrm{mV}$ could be observed on all three surfaces for the peak at $+0.5 \mathrm{~V}$, which moved around $+0.7 \mathrm{~V}$. Alongside this shift, a substantial decrease in the peak intensity was observed. A secondary reduction peak could be observed at around $+0.45 \mathrm{~V}$, when testing the VAN-containing solutions. This second reduction peak is obtained only at a high concentration of VAN and it could be attributed to the reduction of the oxidized form of VAN or to the reduction of a different type of Au found at the surface of the electrode. In the oxidation, a peak at around $+0.9 \mathrm{~V}$ was also observed due to the electrooxidation of VAN (Figure 2).
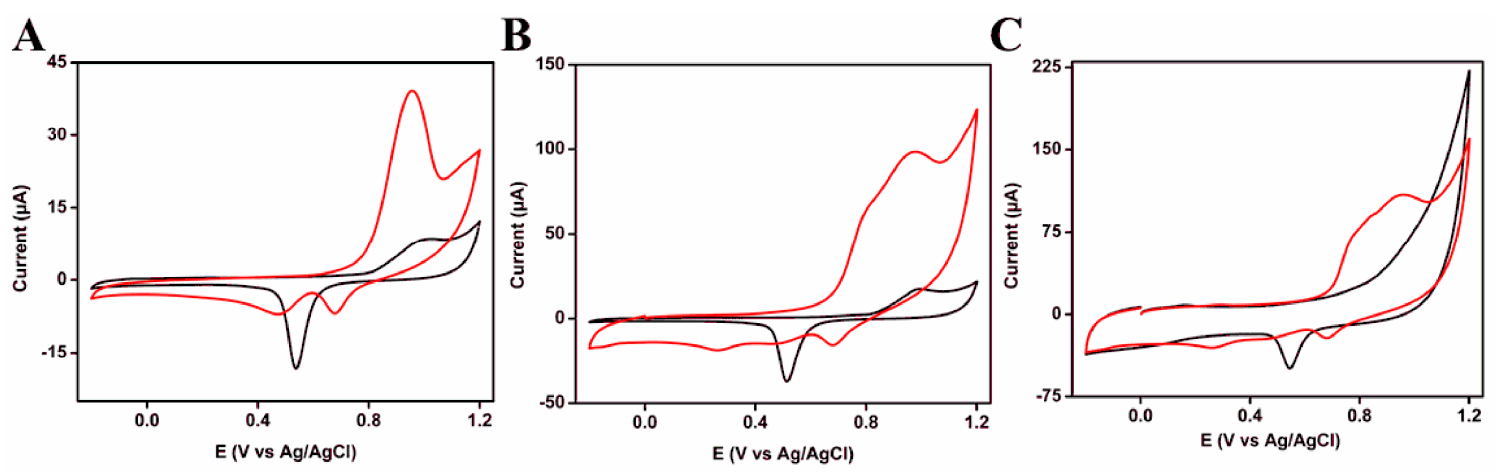

Figure 2. $\mathrm{CVs}$ of $1 \mathrm{mM}$ VAN (red) in $0.1 \mathrm{M} \mathrm{H}_{2} \mathrm{SO}_{4}$ and the blank $\left(0.1 \mathrm{M} \mathrm{H}_{2} \mathrm{SO}_{4}\right)$ (black) solutions at AuNP-C-SPE (A), at AuNS-C-SPE (electrogenerated) (B), and at AuNS-G-SPE (C); Scan rate $0.1 \mathrm{~V} \cdot \mathrm{s}^{-1}$. 
These tests revealed that there is an interaction between the Au and VAN, which manifests both in the oxidation and in the reduction domain. The electrooxidation of VAN is favored by the increase in area of the active surface due to the deposition of AuNSs, while in reduction, the electrochemical signal of Au deposited on the electrode surface is hindered by the physical and chemical interactions between VAN and Au. This interaction is further favored by the graphene-modified electrode surface, which increases the adsorption of VAN onto the sensing surface.

Considering that in oxidation, on the AuNS-G-SPE, no peak was observed for the blank solution and for the VAN solution, the highest current was obtained on AuNS-G-SPE, this surface proved to be the most suitable one. Also, in reduction, the highest decrease in the intensity of the peak was observed for the AuNS-G-SPE.

The CV analysis showed a stronger electrochemical signal for the interaction of VAN-Au in the case of C-SPE electrochemically modified with AuNS (AuNS-C-SPE), compared to the AuNPs-C-SPE, modified with AuNPs by the manufacturer. This proved that the "homemade" modification, on unmodified C-SPE provided a more sensitive platform, with a $30 \mathrm{nA}$ decrease for the C-SPE with AuNP "homemade" compared to $12 \mathrm{nA}$ for AuNPs-C-SPE. Another advantage of doing the modification by ourselves was the ability to better control the modification of the surface.

Literature data suggests that VAN shows a good affinity towards graphene, graphene being used in the procedures for the VAN extraction $[13,15]$. Also, graphene has special properties which make it an interesting working surface in electrochemistry. This direction was also supported by previously reported data [9], which showed that graphene is a more sensitive electrochemical platform for VAN detection and by other data present in the literature [10]. The further improvement of graphene as a sensing platform by modifying it with AuNSs was considered and tested.

The experimental data confirmed the theoretical assumptions showing a similar electrochemical response on both electrodes (AuNS-G-SPE and AuNPs-C-SPE) but with both a higher oxidation peak and a bigger difference between the peak of the blank and the peak of the VAN solution, in reduction, on the AuNS-G-SPE.

\subsubsection{UV-VIS Studies}

Spectrophotometric studies were also carried out to investigate the interaction between VAN and different species of $\mathrm{Au}$ and different nanostructures of $\mathrm{Au}$, in aqueous solutions of $0.1 \mathrm{M} \mathrm{H}_{2} \mathrm{SO}_{4}$. For this, the UV-Vis spectra of VAN and mixtures of VAN and $\mathrm{HAuCl}_{4}$ or AuNPs of different sizes (15 and $50 \mathrm{~nm}$ ) were recorded.

As can be seen in Figure 3, no changes in the spectra were observed for the mixture of VAN and $\mathrm{HAuCl}_{4}$ in fresh solutions, compared to the monocomponent solutions. However, after $4 \mathrm{~h}$, the appearance of a new, smaller peak at around $450 \mathrm{~nm}$ revealed the presence of AuNP, caused by the chemical interaction between VAN and $\mathrm{HAuCl}_{4}$. The $4 \mathrm{~h}$ incubation time was the shortest duration with visible enough differences between the solutions containing VAN and the controls containing only one component. The spectra of the mixtures and of the controls were monitored up to $24 \mathrm{~h}$, the differences between the mixtures and controls, increasing slightly with the increase in the incubation period, indicating the slow kinetics of the interactions in normal conditions.

This data suggested that there is indeed an interaction between VAN and $\mathrm{HAuCl}_{4}$, but which in our working conditions will require a longer period to lead to the formation of a detectable amount of VAN-AuNPs interactions.

When the mixture of VAN and citrate-stabilized AuNPs was tested, for the fresh solutions, small changes, which intensified after $4 \mathrm{~h}$, were observed, indicating the interaction between VAN and AuNPs. The changes were similar for both types of AuNPs, of 15 and $50 \mathrm{~nm}$ diameter. The changes consisted mostly of a shift of the peak of absorption to a longer wavelength, a bathochromic shift. The bathochromic shift arises due to the presence of vancomycin on the surface of gold nanoparticles and possibly in a smaller part, due to a little aggregation of the AuNPs after conjugation with vancomycin. This batochromic shift has been previously reported for the conjugation of vancomycin with AuNPs, 
being an indication of a successful conjugation [17]. These findings confirmed the electrochemical data obtained regarding the VAN-Au interaction.
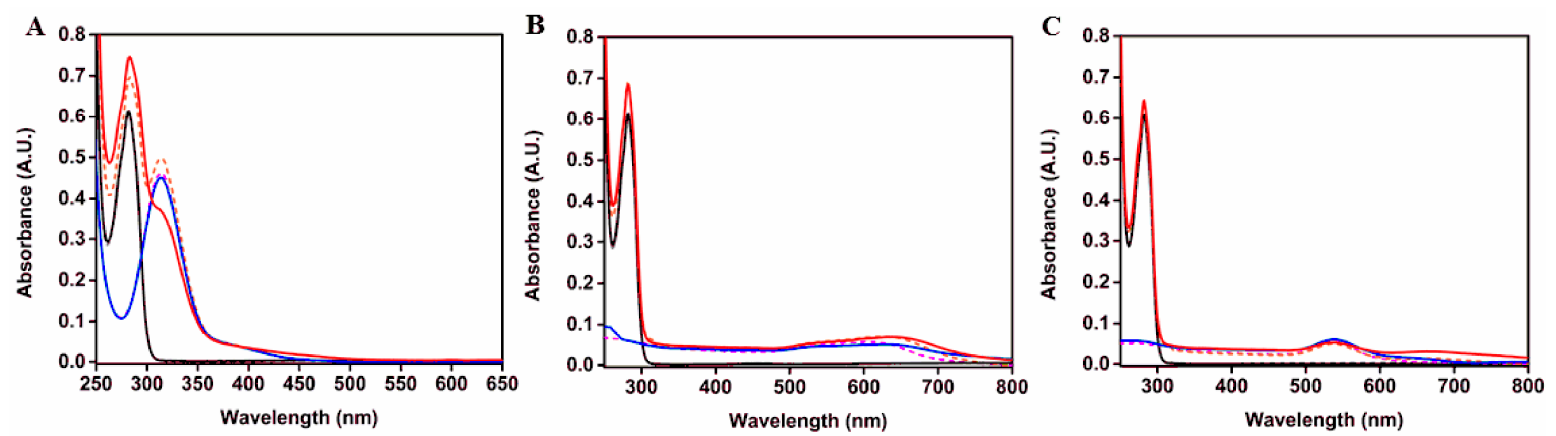

Figure 3. UV-VIS spectra, fresh solutions and after $4 \mathrm{~h}$ of: VAN $0.1 \mathrm{mM}$ (dotted grey and black), Au species ((A) $\mathrm{HAuCl}_{4} 0.1 \mathrm{mM}$, (B) AuNPs $50 \mathrm{~nm}$ and (C) AuNPs $15 \mathrm{~nm}$ ) (dotted pink and blue), and mixture of VAN $0.1 \mathrm{mM}$ and Au species Au species ((A) $\mathrm{HAuCl}_{4} 0.1 \mathrm{mM}$, (B) AuNPs $50 \mathrm{~nm}$ and (C) AuNPs $15 \mathrm{~nm}$ ) (dotted orange and red).

\subsection{Optimization of the Sensing Platform}

\subsubsection{Optimization of the Modification with AuNS}

Once it was established that G-SPEs are the best analytical platform, two different approaches of modifying the sensing surface were investigated in order to obtain the conditions leading to large, well-defined anodic peak for VAN and to the highest, quantifiable difference between the cathodic peak of the blank and in the presence of VAN. The first approach involved the electrogeneration of the AuNSs, using two different techniques, CV and CA. The second approach involved the modification with already synthesized AuNPs, of two different sizes, 15 and $50 \mathrm{~nm}$, through drop-casting.

From the data obtained (Figure 4), the protocol involving the electrogeneration through CA proved to be the best approach, showing a higher sensitivity towards VAN, considering both domains, in oxidation and reduction (blue line, Figure $4 \mathrm{~A}, \mathrm{C}$ ). It could be observed that the sensing surfaces modified through electrogeneration with AuNS were more sensitive towards VAN in reduction compared to the surfaces modified with already synthesized AuNPs, through drop-casting. This can be explained by the higher reactivity of the AuNS obtained in situ, electrochemically, which are not modified on the surfaces with stabilizing anions like citrate, making their physico-chemical interactions with VAN molecules stronger. Also, it can be observed that they present a stronger signal in reduction when the blank solution is tested, due to their higher electrochemical reactivity.

In oxidation, the electrode modified with $15 \mathrm{~nm}$ AuNPs presented an anodic peak for VAN with a peak current a little bigger than the one obtained for the electrode modified with $50 \mathrm{~nm}$ AuNPs. Similarly, a larger anodic peak was obtained for the electrode modified through CA, compared to $\mathrm{CV}$. Even though in oxidation it seemed that the electrodes modified through drop-casting gave slightly better results, considering their worse results in reduction, $10 \mathrm{nA}$ difference compared to $28 \mathrm{nA}$ difference for the electrogenerated AuNSs, the electrogeneration protocol looked to be the more suitable approach overall.

Considering all these, we chose to continue on the sensing platform obtained through electrogeneration by using $C A$. 
A

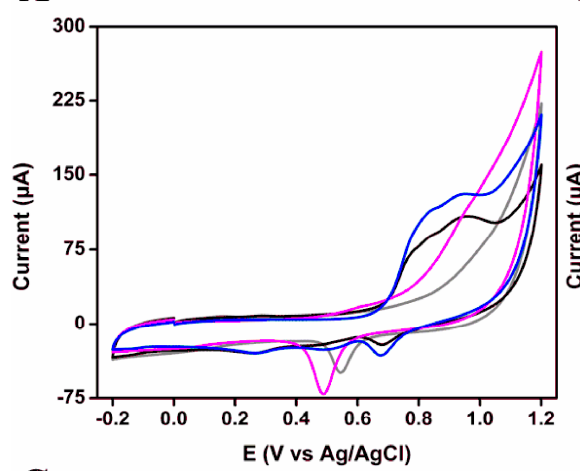

C

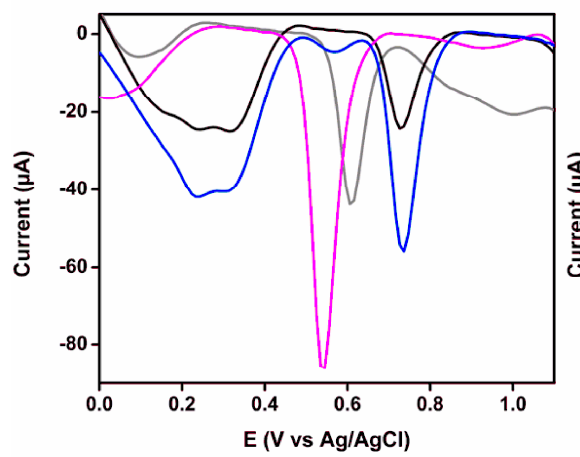

B

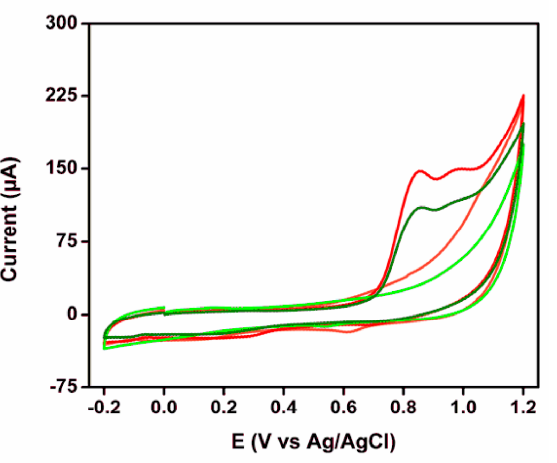

D

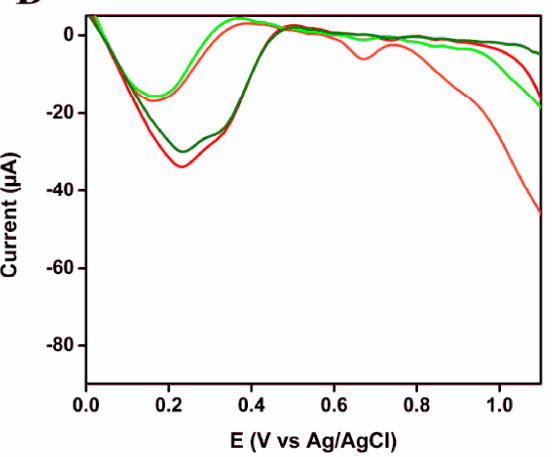

Figure 4. (A) CVs and (C) DPVs of $1 \mathrm{mM}$ VAN in $0.1 \mathrm{M} \mathrm{H}_{2} \mathrm{SO}_{4}$ and the blank solutions at CV-AuNS-G-SPE (black and grey) and CA-AuNS-G-SPE (blue and pink); (B) CVs and (D) DPVs of $1 \mathrm{mM}$ VAN in $0.1 \mathrm{M} \mathrm{H}_{2} \mathrm{SO}_{4}$ and the blank solutions at AuNP $15 \mathrm{~nm} \mathrm{G-SPE} \mathrm{(red} \mathrm{and} \mathrm{orange)} \mathrm{and} \mathrm{AuNP}$ $50 \mathrm{~nm}$ G-SPE (dark green and light green).

\subsubsection{Optimization of the Electrogeneration Procedure}

The potential and time used for the electrogeneration of AuNS were also optimized (Table 1). For the optimization of these parameters, a balance between the signal in the two approaches in oxidation and reduction was considered. For this, both a well-defined peak, clearly distinguishable from the signal of the blank, in oxidation and the biggest difference between the signals of AuNSs in the blank and in the presence of VAN, in reduction, were chosen as selection criteria.

Several potentials from $-0.5 \mathrm{~V}$ up to $0 \mathrm{~V}$ were tested. The experiments showed an increase in the sensitivity with the decrease of the potential applied down to around $-0.4 \mathrm{~V}$. No significant improvement was observed at lower potentials and at higher potentials; a decrease in the response and even a change in the behavior were observed around $0 \mathrm{~V}$, concluding that a potential of $-0.4 \mathrm{~V}$ is the optimal one for the electrogeneration of AuNSs.

Another key parameter in the optimization of the used procedure is the duration of the electrogeneration procedure. Similar to the optimization of the potential, several durations were tested, from 60 up to $600 \mathrm{~s}$. As expected, with the increase of the deposition duration, an increase of the cathodic peak for the blank solution was obtained, due to a higher amount of AuNSs produced onto the electrode surface. This did not translate into an increase in sensitivity. The difference between the AuNSs signal in the blank and the one in the presence of VAN did not increase with the deposition time. In the end, a duration of $120 \mathrm{~s}$ for the deposition procedure proved to be optimal considering that longer deposition durations did not translate to a higher sensitivity. 
Table 1. Optimization of the electrogeneration procedure.

\begin{tabular}{|c|c|c|c|c|}
\hline \multicolumn{5}{|c|}{ Deposition Potential Optimization } \\
\hline \multirow{3}{*}{ Deposition Potential (V) } & \multicolumn{3}{|c|}{ Reduction } & Oxidation \\
\hline & \multicolumn{3}{|c|}{ Response Intensity $(\mu \mathrm{A})$} & Response Intensity ( $\mu \mathrm{A})$ \\
\hline & Blank & $50 \mu \mathrm{M}$ VAN & $I_{\mathrm{B}}-I_{\mathrm{VAN}}$ & $50 \mu \mathrm{M}$ VAN \\
\hline-0.5 & -94.14 & -55.30 & -38.84 & 43.87 \\
\hline-0.4 & -89.04 & -48.89 & -40.13 & 45.43 \\
\hline-0.3 & -77.67 & -40.92 & -36.75 & 42.35 \\
\hline-0.2 & -66.84 & -42.21 & -24.63 & 40.90 \\
\hline-0.1 & -51.45 & -46.8 & -4.65 & 37.16 \\
\hline 0 & -36.57 & -42.59 & 6.02 & 32.92 \\
\hline \multicolumn{5}{|c|}{ Deposition Time Optimization } \\
\hline \multirow{3}{*}{ Deposition Time (s) } & & Reduction & & Oxidation \\
\hline & \multicolumn{3}{|c|}{ Response Intensity $(\mu \mathrm{A})$} & Response Intensity $(\mu \mathrm{A})$ \\
\hline & Blank & $50 \mu \mathrm{M}$ VAN & $I_{\mathrm{B}}-I_{\mathrm{VAN}}$ & $50 \mu \mathrm{M}$ VAN \\
\hline 60 & -54.91 & -39.86 & -15.05 & 61.00 \\
\hline 120 & -89.04 & -48.89 & -40.13 & 45.43 \\
\hline 240 & -89.69 & -73.48 & -16.21 & 44.67 \\
\hline 480 & -127.33 & -98.49 & -28.84 & 41.98 \\
\hline 600 & -174.29 & -138.67 & -35.62 & 37.22 \\
\hline
\end{tabular}

$I_{\mathrm{B}}$ - the current recorded for the blank, $I_{\mathrm{VAN}}{ }^{-}$the current recorded in the presence of VAN.

\subsubsection{Optimization of the $\mathrm{pH}$}

Another optimized parameter was the $\mathrm{pH}$ used for detection. For this, $\mathrm{H}_{2} \mathrm{SO}_{4} 0.1 \mathrm{M}, \mathrm{pH} 1$ and PBS $50 \mathrm{mM}$ adjusted to different values of $\mathrm{pH}$, from 2 to 12, were tested as supporting electrolyte solutions for VAN detection (Figure 5).

A

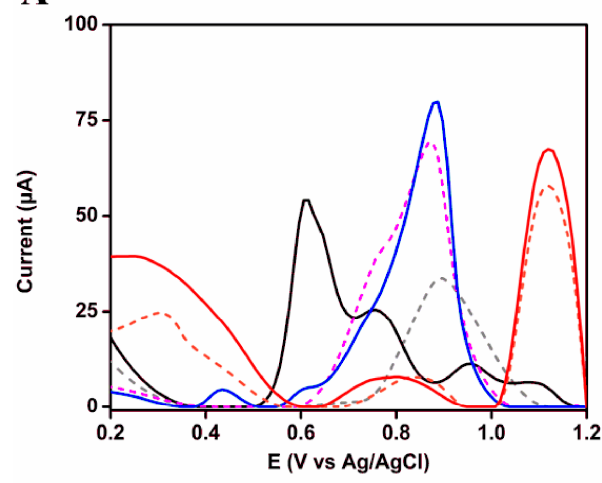

B

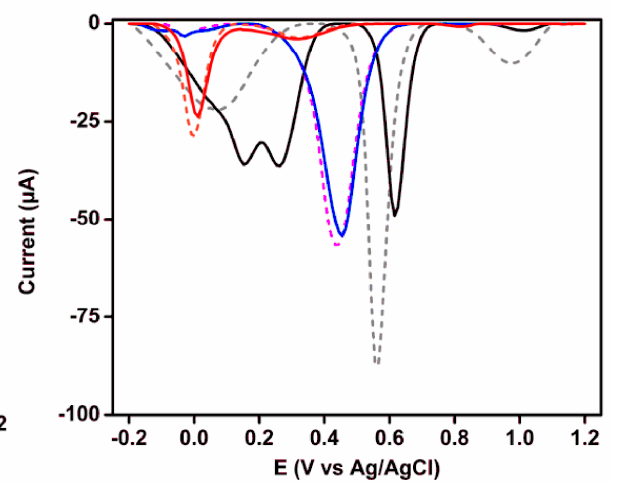

Figure 5. DPVs in (A) oxidation and (B) reduction of $50 \mu \mathrm{M}$ VAN and the blank solutions at CA-AuNS-G-SPE at pH 1 (black and dotted grey), pH 7 (blue and dotted pink) and pH 12 (red and dotted orange).

It could be observed that $\mathrm{H}_{2} \mathrm{SO}_{4} 0.1 \mathrm{M}$, pH 1 gave the best results, for both detection approaches, direct in oxidation and also indirect in reduction (Figure 5). For the detection in oxidation, a clearly defined peak could be observed, in comparison to the signal of the blank solution, only for the acidic medium.

Regarding the indirect detection in reduction, the biggest difference between the signal of the blank solution and the one containing VAN was observed for the $0.1 \mathrm{M} \mathrm{H}_{2} \mathrm{SO}_{4}$ solution. This result 
can be explained by the higher electroactivity at lower $\mathrm{pH}$ of the AuNSs, found at the surface of the electrode and by the higher affinity of VAN towards AuNSs at this low $\mathrm{pH}$.

\subsection{Analytical Performance}

\subsubsection{Calibration Curves}

Two calibration curves were realized for each domain of detection in the range of concentrations from 1 to $100 \mu \mathrm{M}$ VAN (Figure 6). The linear relationships found, with their correlated equations and their correlation coefficients $\left(R^{2}\right)$, were as follows: $I_{\text {VAN }}(\mu \mathrm{A})=0.7689 \times C_{\text {VAN }}(\mu \mathrm{M})-0.0332$; with $R^{2}=0.9873$, for the oxidation domain and, $I_{\mathrm{B}}-I_{\mathrm{VAN}}(\mu \mathrm{A})=-0.3782 \times C_{\mathrm{VAN}}(\mu \mathrm{M})-18.791$; with $R^{2}=0.9713$, for the reduction domain.

For the determination of the limit of detection (LOD), the following equation was used: LOD $=3 \times S b / m$, in which $S b$ is the standard deviation of the response of the blank solution and $m$ is the slope of the calibration curve. A LOD of 0.29 and $0.5 \mu \mathrm{M}$ was obtained for the oxidation domain and the reduction domain, respectively.
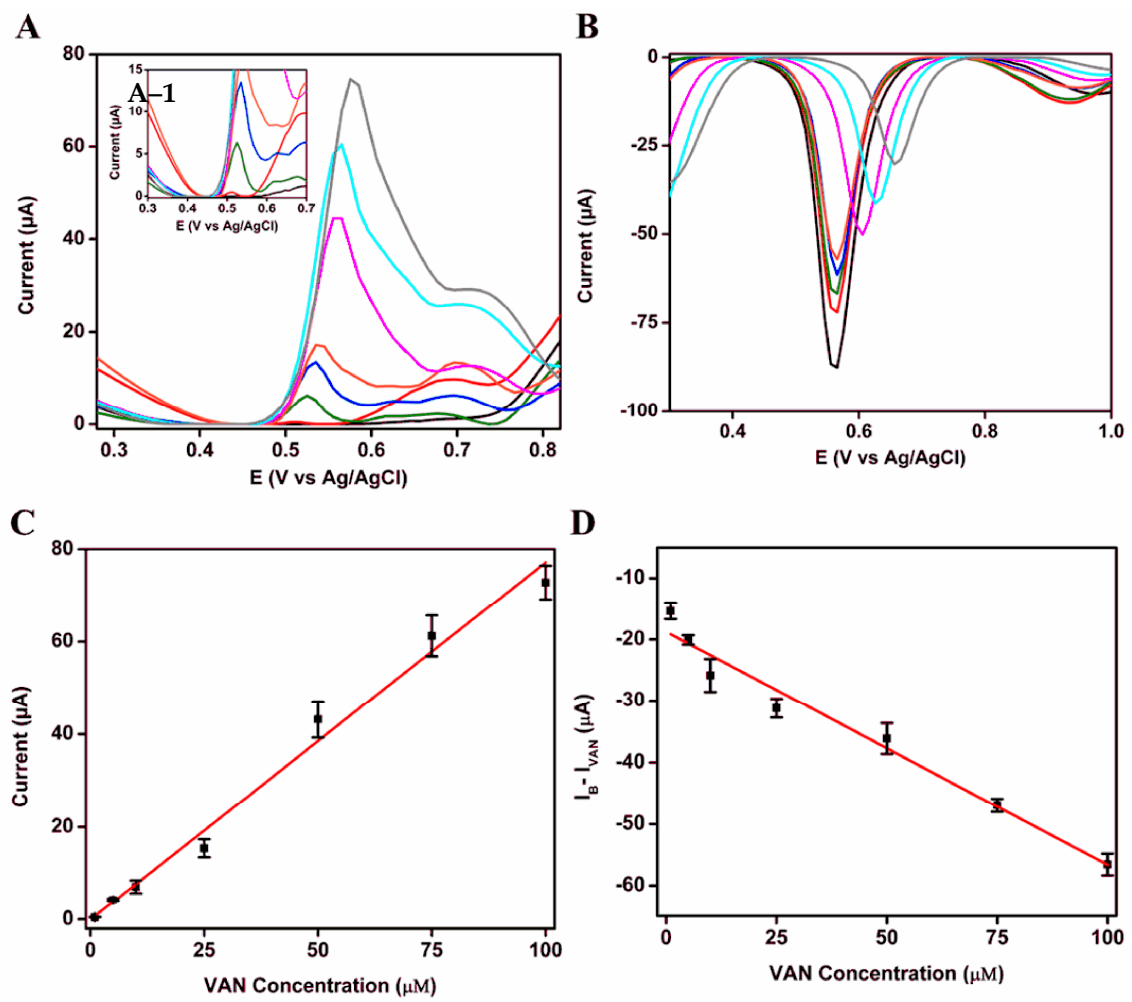

Figure 6. (First row) DPVs in oxidation (A) and in reduction (B) at AuNS-G-SPE of $1 \mu \mathrm{M}$ (red), $5 \mu \mathrm{M}$ (green), $10 \mu \mathrm{M}$ (blue), $25 \mu \mathrm{M}$ (orange), $50 \mu \mathrm{M}$ (pink), $75 \mu \mathrm{M}$ (cyan), and $100 \mu \mathrm{M}$ (grey) vancomycin solution in $0.1 \mathrm{M} \mathrm{H}_{2} \mathrm{SO}_{4}$ (blank) ((A-1) - zoomed area); (Second row) Linear relationship between the response in oxidation (C) and the response in reduction (D) for VAN concentration from 1 to $100 \mu \mathrm{M}$.

\subsubsection{Selectivity Studies}

The selectivity of the method was tested by recording the signal of VAN in mixture solutions, with other antibiotics and drugs. Both approaches proved to be selective towards VAN, its signal not being significantly affected by the presence of the tested interferents (Figure 7). 
A

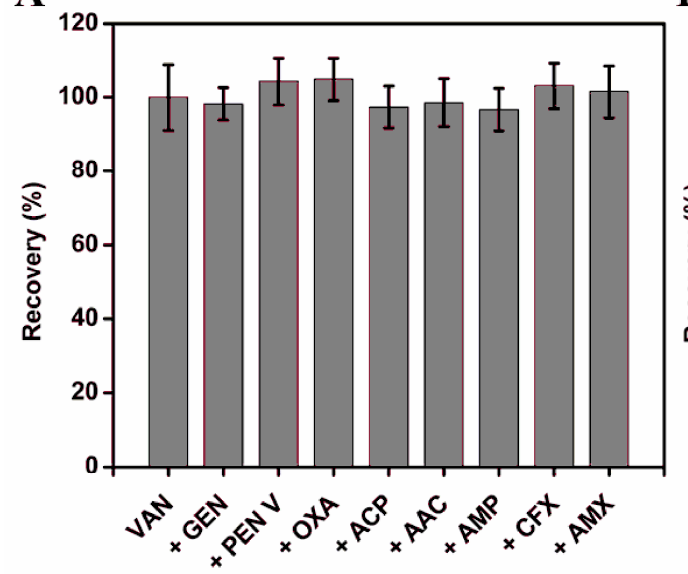

B

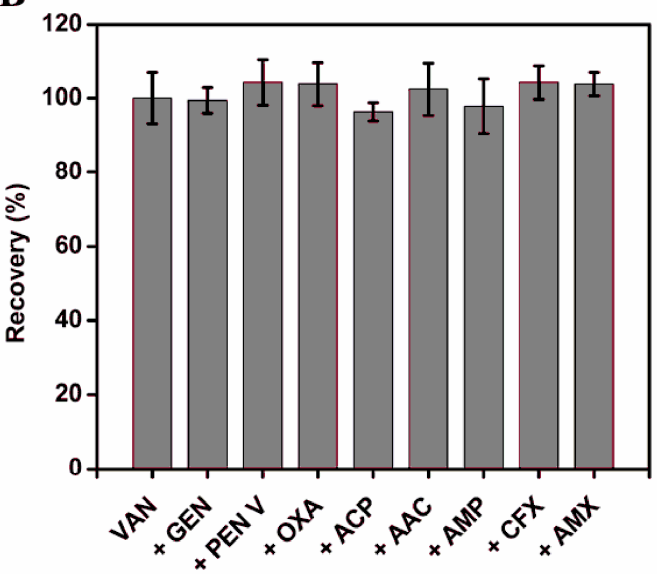

Figure 7. Selectivity tests using the optimized method for direct approach in oxidation (A) and for the indirect approach in reduction (B).

\subsubsection{Real Sample Analysis}

The developed method was tested by analyzing commercially available human serum of real samples, containing VAN (Figure 8). The concentration of VAN in the tested samples was determined using the standard addition method. Recoveries of $97.38 \%$ and $104.54 \%$ in oxidation and of $107.06 \%$ and of $103.70 \%$ in reduction were obtained for both approaches after a minimal sample treatment.
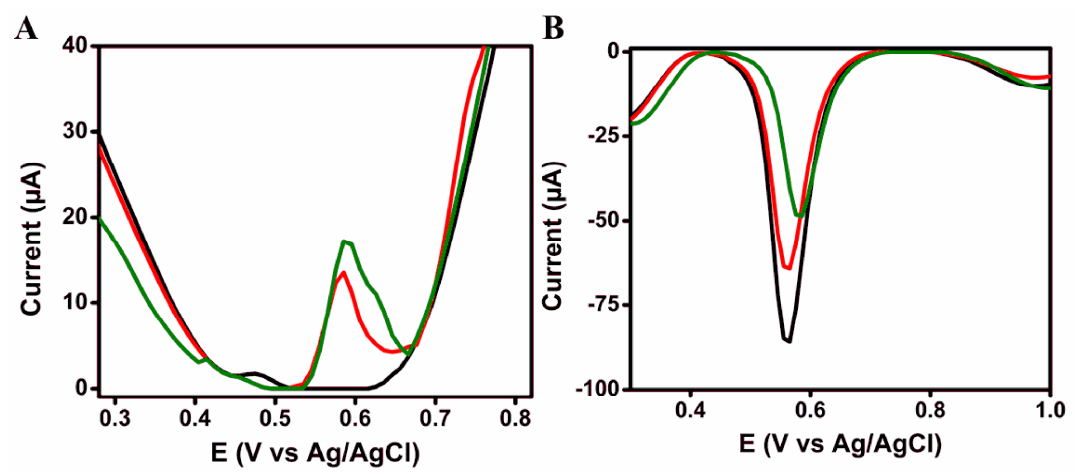

Figure 8. Real sample analysis: (A) in oxidation and (B) in reduction of blank diluted serum (black) and diluted serum spiked with $10 \mu \mathrm{M}$ (red) and $50 \mu \mathrm{M}$ (green) final concentrations of VAN.

\section{Conclusions}

A selective method was developed for the electrochemical analysis of VAN, based on a SPE modified with a hybrid graphene-Au nanocomposite with two different detection approaches in one analysis, one direct using the electrooxidation of VAN, and the other one indirect, based on the intrinsic electrochemical signal of AuNS, in reduction, and their interaction with VAN.

This study shows the utility of the combination of two different nanomaterials such as graphene and AuNS and how they can be combined in an electroanalytical platform for the sensitive detection of an antibiotic.

Author Contributions: Conceptualization, A.B., B.F., A.-M.D. and A.P.; methodology, A.B. and B.F.; validation, B.F. and C.C.; investigation, A.B., A.-M.D. and A.P.; data curation, A.B. and A.P.; writing-original draft preparation, A.B.; writing-review and editing, A.B., B.F. and C.C.; supervision, C.C.; project administration, C.C. and B.F.; funding acquisition, C.C., B.F. and A.B.

Funding: This work was supported by grants from the Ministry of Research and Innovation, CNCS - UEFISCDI, project number PN-III-P1-1.1-PD-2016-1132 and project RusEraNet ST PLASMON ELECTROLIGHT, within 
PNCDI III. This work was also supported by a PhD Research Project no. PCD 1680/26/19.01.2018 offered by University of Medicine and Pharmacy "Iuliu Hatieganu", Cluj-Napoca.

Conflicts of Interest: The authors declare no conflict of interest.

\section{References}

1. Bruniera, F.R.; Ferreira, F.M.; Saviolli, L.R.M.; Bacci, M.R.; Feder, D.; Pedreira, M.D.L.G.; Peterlini, M.A.S.; Azzalis, L.; Junqueira, V.B.C.; Fonseca, F.L. The use of vancomycin with its therapeutic and adverse effects: A review. Eur. Rev. Med. Pharmacol. Sci. 2015, 19, 694-700. [PubMed]

2. Rubinstein, E.; Keynan, Y. Vancomycin revisited-60 years later. Front. Public Health 2014, 2, 1-7. [CrossRef] [PubMed]

3. World Health Organization. Antimicrobial Resistance: Global Report on Surveillance. Available online: http://www.who.int/drugresistance/documents/surveillancereport/en/ (accessed on 18 August 2019).

4. World Health Organization. WHO Guidelines on Use of Medically Important Antimicrobials in Food-Producing Animals. Available online: http://www.who.int/antimicrobial-resistance/en/ (accessed on 18 August 2019).

5. World Health Organization. Tackling Antibiotic Resistance from a Food Safety Perspective in Europe. Available online: http://www.euro.who.int/en/publications/abstracts/tackling-antibiotic-resistance-from-afood-safety-perspective-in-europe (accessed on 18 August 2019).

6. Nagel, O.G.; Beltrán, M.C.; Molina, M.P.; Althaus, R.L. Novel microbiological system for antibiotic detection in ovine milk. Small Rumin. Res. 2012, 102, 26-31. [CrossRef]

7. Javorska, L.; Krcmova, L.K.; Solichova, D.; Solich, P.; Kaska, M. Modern methods for vancomycin determination in biological fluids by methods based on high-performance liquid chromatography-A review. J. Sep. Sci. 2016, 39, 6-20. [CrossRef] [PubMed]

8. Sanvicens, N.; Mannelli, I.; Salvador, J.P.; Valera, E.; Marco, M.P. Biosensors for pharmaceuticals based on novel technology. TrAC Trends Anal. Chem. 2011, 30, 541-553. [CrossRef]

9. Feier, B.; Blidar, A.; Vlase, L.; Cristea, C. The complex fingerprint of vancomycin using electrochemical methods and mass spectrometry. Electrochem. Commun. 2019, 104, 106474. [CrossRef]

10. Hadi, M.; Mollaei, T. Electroanalytical determination of vancomycin at a graphene-modified electrode: Comparison of electrochemical property between graphene, carbon nanotube, and carbon black. Electroanalysis 2018, 31, 1224-1228. [CrossRef]

11. Gandouzi, I.; Tertis, M.; Cernat, A.; Bakhrouf, A.; Coros, M.; Pruneanu, S.; Cristea, C. Sensitive detection of pyoverdine with an electrochemical sensor based on electrochemically generated graphene functionalized with gold nanoparticles. Bioelectrochemistry 2018, 120, 94-103. [CrossRef] [PubMed]

12. Fritea, L.; Le Goff, A.; Putaux, J.L.; Tertis, M.; Cristea, C.; Săndulescu, R.; Cosnier, S. Design of a reduced-graphene-oxide composite electrode from an electropolymerizable graphene aqueous dispersion using a cyclodextrin-pyrrole monomer. Application to dopamine biosensing. Electrochim. Acta 2015, 178, 108-112. [CrossRef]

13. Radu, E.; Stoica, R.; Doncea, S.M.; Vasilievici, G.; Oprescu, E.E.; Bolocan, I.; Ogaidi, A.G.M.A.; Ion, I.; Ion, A.C. Vancomycin sorption on pristine and oxidized exfoliated graphite nanoplatelets. Rev. Chim. 2016, 67, 401-407.

14. Peng, J.; Feng, Y.; Han, X.X.; Gao, Z.N. Sensitive electrochemical detection of melamine based on gold nanoparticles deposited on a graphene doped carbon paste electrode. Anal. Methods 2016, 8, $2526-2532$. [CrossRef]

15. Khalilian, F.; Hanzaki, S.A.; Yousefi, M. Synthesis of a graphene-based nanocomposite for the dispersive solid-phase extraction of vancomycin from biological samples. J. Sep. Sci. 2015, 38, 975-981. [CrossRef] [PubMed]

16. Hur, Y.E.; Park, Y. Vancomycin-functionalized gold and silver nanoparticles as an antibacterial nanoplatform against methicillin-resistant staphylococcus aureus. J. Nanosci. Nanotechnol. 2016, 16, 6393-6399. [CrossRef] [PubMed]

17. Fayaz, A.M.; Girilal, M.; Mahdy, S.A.; Somsundar, S.S.; Venkatesan, R.; Kalaichelvan, P.T. Vancomycin bound biogenic gold nanoparticles: A different perspective for development of anti VRSA agents. Process. Biochem. 2011, 6, 636-641. [CrossRef] 
18. Wang, S.G.; Chen, Y.C.; Chen, Y.C. Antibacterial gold nanoparticle-based photothermal killing of vancomycin-resistant bacteria. Nanomedicine 2018, 13, 1405-1416. [CrossRef] [PubMed]

19. Hezard, T.; Fajerwerg, K.; Evrard, D.; Collière, V.; Behra, P.; Gros, P. Influence of the gold nanoparticles electrodeposition method on $\mathrm{Hg}(\mathrm{II})$ trace electrochemical detection. Electrochim. Acta 2012, 73, 15-22. [CrossRef]

20. Tertiș, M.; Cernat, A.; Lacatiș, D.; Florea, A.; Bogdan, D.; Suciu, M.; Săndulescu, R.; Cristea, C. Highly selective electrochemical detection of serotonin on polypyrrole and gold nanoparticles-based 3D architecture. Electrochem. Commun. 2017, 75, 43-47. [CrossRef]

(C) 2019 by the authors. Licensee MDPI, Basel, Switzerland. This article is an open access article distributed under the terms and conditions of the Creative Commons Attribution (CC BY) license (http://creativecommons.org/licenses/by/4.0/). 